\title{
EFFECTIVENESS OF A CARE HOME MENTAL HEALTH LIAISON (CHL) SERVICE IN A SCOTTISH HEALTH REGION
}

\author{
Gary Stevenson \& Tracy McEwan \\ Department of Psychiatry, NHS Fife, Fife, Scotland KY15 5RR (gstevenson@nhs.net)
}

\section{Background \& Objectives}

- The Fife CHL service was established in late 2017 funded by the Scottish Government's Primary Care Transformation Fund, recognising the demands on Primary Care (GP) services from care home populations with mental health problems. Specialist mental health input to Care Homes prior to $\mathrm{CHL}$ service development was variable and lacked equity of provision in this region.

- To develop and report on the effectiveness of the CHL service for the 3000 care home residents within the 75 Care Homes within a Scottish Health region (Fife, population $365 \mathrm{k}$ ), to reduce their psychiatric admissions and to reduce levels of antipsychotic prescribing. To provide training and education in a modular format to Care Home staff to allow them to better understand, recognise and prevent stress and distressed behaviours in people with dementia

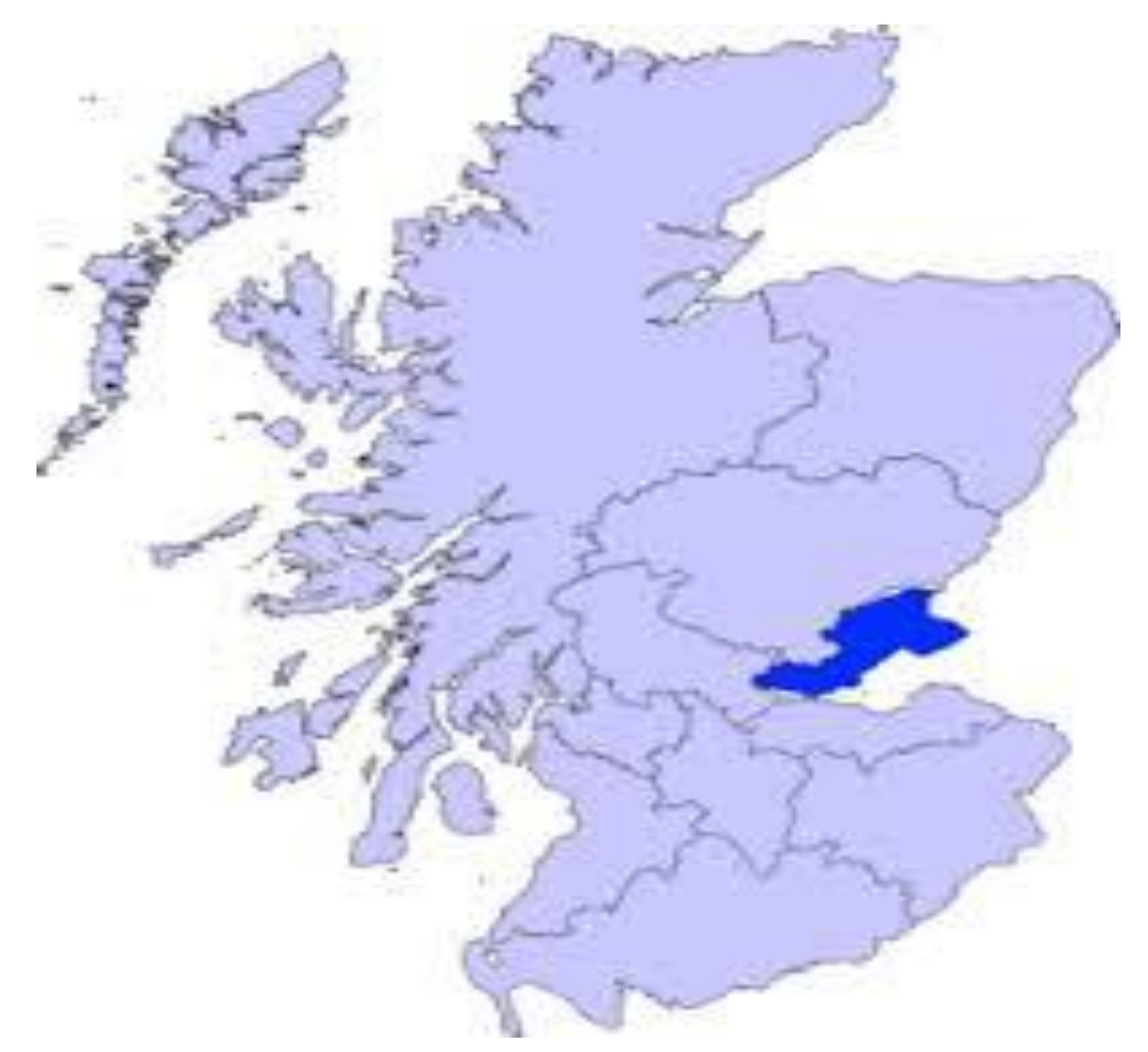

\section{Methods}

Data were collected prospectively on demographics, referral patterns, diagnoses, response times, clinical recommendations and outcomes. Satisfaction surveys of Care Home staff, relatives of and persons with dementia, and General Practitioners (GPs) were undertaken.

\section{Results}

During 2018, 524 new referrals (73\% dementia diagnoses) were seen within operational guidelines ( 3 days for urgent, 10 days for routine), with an average of 225 nursing visits per month.

For residents with dementia prescribed antipsychotics, $76 \%$ received individualised consultant recommendations to either reduce and/or discontinue the antipsychotics with a potential pharmacy saving of $£ 30 \mathrm{k} / \mathrm{year}$.

$41 \%$ reduction in admissions from Care Homes to psychiatric wards occurred when compared to the pre-CHL year.

95 staff from 21 different care home establishments had a total of 205 educational session attendances

Feedback from surveys identified an overall positive experience of interactions with the $\mathrm{CHL}$ team, as well as areas for further service development

\section{Conclusions}

- Development of the CHL service allowed a focussed, responsive service that achieved its primary objectives of service equity, reducing psychiatric admissions and reducing antipsychotic prescribing.

- All of the GPs who responded to the survey reported that the CHL service added benefit compared to previous service provision.

- The prompt response to referrals and the achievement of CHL service objectives, suggests patients are being seen in their own home, by the right person and at the right time. 